\title{
Hyperperfusion Syndrome After Clipping an Unruptured Cerebral Aneurysm
}

\author{
-Two Case Reports-
}

\author{
Toshiya Sugino*,**, Masafumi OHTAKI*, Masahiko WANiBuChI**, \\ Sonen KIN*, and Kiyohiro HOUKIN** \\ * Department of Neurosurgery, Obihiro Kosei General Hospital, Obihiro, Hokkaido; \\ **Department of Neurosurgery, Sapporo Medical University, Sapporo, Hokkaido
}

\begin{abstract}
A 68-year-old woman presented with severe headache 9 days after undergoing successful clipping of a right middle cerebral artery aneurysm. Postoperative imaging revealed increased perfusion and diffuse edema in the right frontotemporal cortex. A 57-year-old woman exhibited perseveration soon after undergoing successful clipping of an anterior communicating artery aneurysm. Postoperative imaging studies revealed increased perfusion and diffuse edema in the left frontal and insular cortex. The symptoms and diffuse edema gradually resolved in both patients. These two cases of hyperperfusion syndrome occurred in a series of 190 patients treated by clipping of unruptured cerebral aneurysms. Hyperperfusion syndrome is a rare complication following aneurysm surgery, especially surgery for unruptured cerebral aneurysms without temporary clipping.
\end{abstract}

Key words: hyperperfusion, clipping, unruptured cerebral aneurysm, single photon emission computed tomography, magnetic resonance imaging

\section{Introduction}

Hyperperfusion syndrome is known to occur after carotid endarterectomy, extracranial-intracranial bypass surgery, and percutaneous transluminal angioplasty. ${ }^{3,8,10,11)}$ Hyperperfusion syndrome is defined as cerebral blood flow (CBF) in excess of the flow required for metabolic needs, and is characterized by the diagnostic triad of unilateral headache, seizures, and intracranial hemorrhage. ${ }^{5)}$ Hyperperfusion syndrome has also been reported following surgery for giant aneurysms after temporary clipping6) and for subarachnoid hemorrhage. ${ }^{2,9)}$ However, hyperperfusion syndrome has rarely been reported after clipping the neck of an unruptured cerebral aneurysm. ${ }^{4)}$

We report two cases of hyperperfusion syndrome in a series of 190 consecutive patients who underwent clipping of unruptured cerebral aneurysms between 2000 and 2008.

\section{Case Reports}

Case 1: A 68-year-old woman was admitted to our hospital with a right middle cerebral artery (MCA) aneurysm. The patient had a history of hypertension and a family history of subarachnoid hemorrhage. Three-dimensional (3D) computed tomography (CT) angiography revealed that the diameter of the aneurysm was $4 \mathrm{~mm}$. Right frontotemporal craniotomy was performed. Opening of the sylvian fissure

Received June 25, 2009; Accepted September 14, 2009 caused minor injury of the superficial sylvian vein. The aneurysm was successfully obliterated with a single clip, after compressing the right temporal lobe with a spatula. Temporary clipping was not performed. Arachnoid plasty using an absorbable collagen sheet hemostat (Instat ${ }^{\circledR}$; Ethicon, Inc., Somerville, N.J., U.S.A.) and fibrin glue (Beriplast ${ }^{\circledR}$ P; CSL Behring, King of Prussia, Pa., U.S.A.) was performed to prevent subdural effusion. No neurological deficits were observed after surgery. The patient complained of unilateral headache on the side of the craniotomy on postoperative day (POD) 1 . CT revealed no hemorrhage and significant edema. The headache was treated with non-steroidal anti-inflammatory drugs. No abrupt elevation of blood pressure was observed postoperatively and the patient's blood pressure remained normotensive.

The patient suffered severe right frontotemporal headache on POD 9. Serial fluid-attenuated inversion recovery (FLAIR) magnetic resonance (MR) imaging obtained on POD 9 demonstrated a hyperintense area in the right frontotemporal cortex (Fig. 1A). 3D time of flight (TOF) MR angiography showed that the right MCA was isointense with the left MCA. Iodine-123 N-isopropyl-p-iodoamphetamine ( ${ }^{123}$ I-IMP) single photon emission computed tomography (SPECT) on POD 13 revealed an approximately $20-30 \%$ increase in the regional CBF ( $\mathrm{rCBF}$ ) of the right frontotemporal lobe compared to the preoperative rCBF (Fig. 2). The patient's blood pressure was monitored closely and remained normotensive. The patient's 

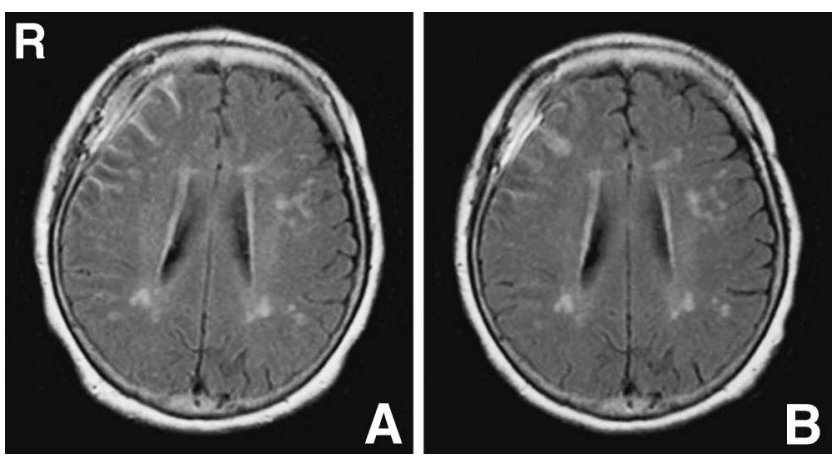

Fig. 1 Case 1. A: Fluid-attenuated inversion recovery (FLAIR) magnetic resonance (MR) image obtained on postoperative day 9 demonstrating a hyperintense area in the right frontotemporal cortex. B: FLAIR MR image obtained on postoperative day 19 demonstrating reduction in the hyperintense area.
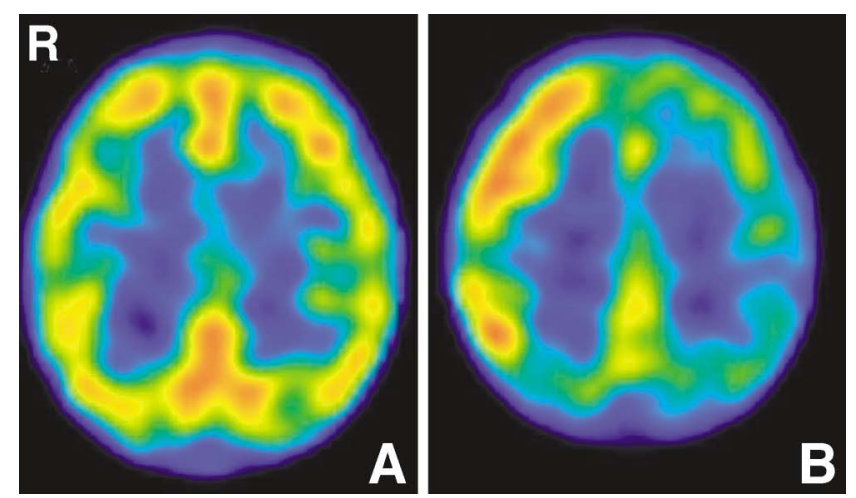

Fig. 2 Case 1. A: Axial iodine-123 N-isopropyl-p-iodoamphetamine ( ${ }^{123}$ I-IMP) single photon emission computed tomography (SPECT) scan obtained before the operation. B: Axial ${ }^{123}$ I-IMP SPECT scan obtained on postoperative day 13 demonstrating increased regional cerebral blood flow in the right frontotemporal lobe.

headache had improved on POD 18, and serial FLAIR MR imaging on POD 19 demonstrated reduction in the hyperintense area (Fig. 1B). ${ }^{123}$ I-IMP SPECT on POD 20 showed the same rCBF as observed preoperatively. The patient had no headache when discharged from the hospital.

Case 2: A 57-year-old woman was admitted to our hospital for an anterior communicating artery aneurysm. 3D CT angiography revealed that the diameter of the aneurysm was $4.5 \mathrm{~mm}$. The patient had a history of craniopharyngioma removed three times through left frontotemporal craniotomy. On admission, the patient's visual acuity was 0.2 on the right, zero on the left, and visual field examination showed right afferent disorder resulting from postoperative sequelae. Left frontotemporal craniotomy was performed to prevent right optic nerve injury. Opening of the sylvian fissure revealed the strongly adherent arachnoid membrane and several fine sylvian veins were sacrificed. The aneurysm was successfully obliterated
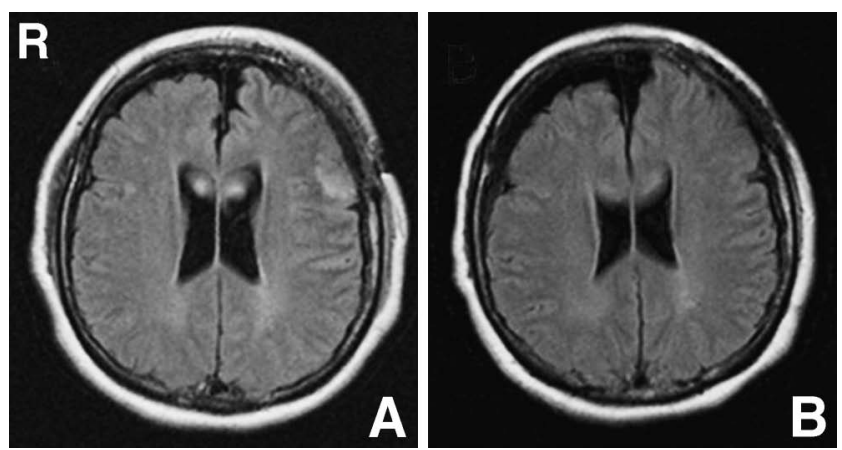

Fig. 3 Case 2. A: Fluid-attenuated inversion recovery (FLAIR) magnetic resonance (MR) image obtained on postoperative day 3 demonstrating hyperintense areas in the left frontotemporal cortex. B: FLAIR MR image obtained on postoperative day 16 demonstrating reduction in the hyperintense areas.
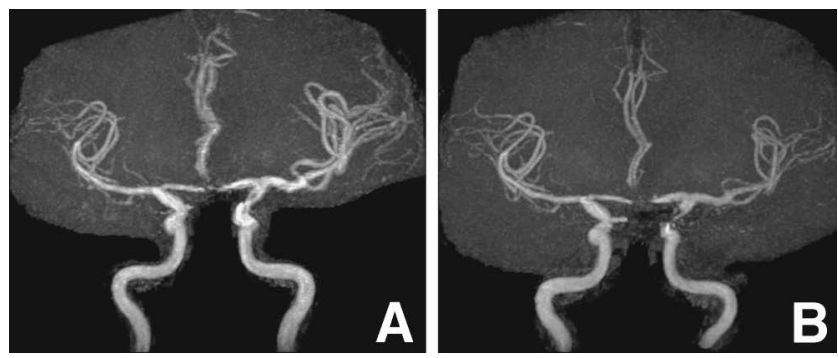

Fig. 4 Case 2. A: Three-dimensional (3D) time of flight (TOF) magnetic resonance (MR) angiogram obtained on postoperative day 8 demonstrating higher intensity of the left middle cerebral artery (MCA) compared to that of the right MCA. B: 3D TOF MR angiogram obtained on postoperative day 17 demonstrating that reduction in the intensity of the left MCA compared to that of the right MCA.
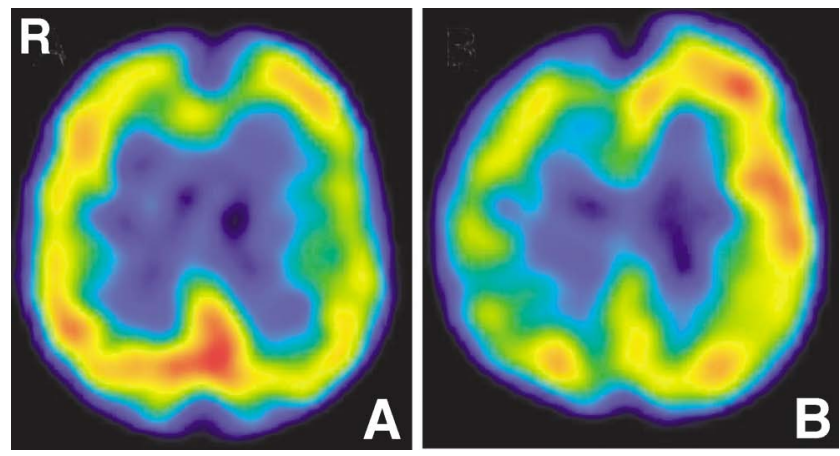

Fig. 5 Case 2. A: Axial iodine-123 $\mathrm{N}$-isopropyl-p-iodoamphetamine (123I-IMP) single photon emission computed tomography (SPECT) scan obtained before the operation. B: Axial ${ }^{123}$ I-IMP SPECT scan obtained on postoperative day 9 demonstrating increased regional cerebral blood flow in the left middle cerebral artery territory. 
with a single clip, after compressing the left frontal lobe with a spatula without temporary clipping. Arachnoid plasty using an absorbable collagen sheet and fibrin glue was performed to prevent subdural effusion.

Soon after the operation, the patient exhibited perseveration and gradually progressed to severe coma. This symptom was not caused by seizure because electroencephalography did not show spike waves. MR imaging obtained immediately after the symptom onset revealed neither infarction nor hemorrhage. However, serial FLAIR MR imaging demonstrated hyperintense areas in the left frontal and insular cortex. No abrupt elevation of blood pressure was observed postoperatively and the patient's blood pressure remained normotensive. Serial FLAIR MR imaging obtained on POD 3 demonstrated enlargement of the hyperintense areas (Fig. 3A). 3D TOF MR angiography obtained on POD 8 showed that increased intensity of the left MCA compared to that of the right (Fig. 4A). ${ }^{123}$ I-IMP SPECT obtained prior to the operation revealed diffuse hypoperfusion, particularly in the left frontal region. However, ${ }^{123}$ I-IMP SPECT obtained on POD 9 showed an approximately $50 \%$ increase in the $\mathrm{rCBF}$ of the left MCA territory compared to before the operation (Fig. 5). The patient remained in a severe coma between POD 1 and POD 9, but recovered to mild confusion on POD 11. Serial FLAIR MR imaging on POD 16 demonstrated reduction in the hyperintense areas (Fig. 3B). 3D TOF MR angiography obtained on POD 17 showed that lower intensity of the left MCA compared to that of the right (Fig. 4B). ${ }^{123}$ I-IMP SPECT obtained on POD 17 showed the same rCBF as in the preoperative study. The patient had cognitive dysfunction when discharged from the hospital.

\section{Discussion}

Patients with hyperperfusion syndrome typically present with severe headache, vomiting, confusion, seizures, and other neurological signs of increased intracranial pressure. Patients with hyperperfusion syndrome may exhibit neurological deficits, such as hemiparesis and aphasia, after undergoing clipping of an unruptured cerebral aneurysm. ${ }^{4,6)}$

Hyperperfusion after clipping of an unruptured cerebral aneurysm has been reported following surgery for giant internal carotid artery aneurysms ${ }^{6)}$ and surgery using a temporary occlusion technique. ${ }^{4)}$ Hyperperfusion may be caused by increased distal blood flow after clipping of a giant aneurysm ${ }^{6}$ or by post-ischemic reperfusion after temporary occlusion of the parent artery. ${ }^{4)}$ In our two cases, the aneurysms were less than $5 \mathrm{~mm}$ in diameter, and temporary clipping was not performed. Therefore, the postoperative hyperperfusion was probably not caused by decreased blood flow and was unrelated to temporary occlusion.

Many factors may be involved in brain ischemia in surgery, including brain compression with a spatula, damage to venous returns, disturbed collateral blood supply, patient's age, operative procedures, and the effects of anesthetic and other agents. ${ }^{1)}$ In our cases, the hyperperfusion syndrome may have been related to the compression with a spatula and injury to the superficial sylvian vein, but the hyperperfusion area did not correspond with the compressed area and did not contain damaged venous returns. Therefore, other causes may be considered for the occurrence of hyperperfusion.

The symptoms and features of our cases are similar to cortical spreading depression (CSD) syndrome. CSD is a self-propagating wave of cellular depolarization that has been implicated in progressive neuronal injury after stroke and head trauma. ${ }^{7}$ Patients with CSD syndrome present with neurological signs of brain edema, such as headache, vomiting, and confusion. The relationship between CSD and blood flow changes remains poorly understood, but experimental studies have shown that local CBF actually increased during CSD. CSD is associated with tissue hypoxia persisting for more than 2 minutes, massive neuronal swelling, and local CBF which actually increases during CSD. ${ }^{12)}$ Local cerebral tissue in rats undergoes hyperperfusion due to arteriolar dilation after the oligemic period of approximately 5 to 8 seconds. ${ }^{13)}$ Such temporary tissue hypoxia may be caused by procedures in clipping and compression by a spatula, so CSD-like waves may be spontaneously elicited.

Hyperperfusion syndrome is a rare complication following surgery for unruptured cerebral aneurysms without temporary clipping. The clinical course and symptoms were different in our two cases, so the causes of the hyperperfusion were probably not the same. Postoperative SPECT and FLAIR MR imaging may be useful in identifying potential cases of hyperperfusion.

\section{References}

1) Andrews RJ, Bringas JR: A review of brain retraction and recommendations for minimizing intraoperative brain injury. Neurosurgery 33: 1052-1064, 1993

2) Dinh YRT, Lot G, Benrabah R, Baroudy O, Cophignon J, Seylaz J: Abnormal cerebral vasodilation in aneurysmal subarachnoid hemorrhage: use of serial ${ }^{133} \mathrm{Xe}$ cerebral blood flow measurement plus acetazolamide to assess cerebral vasospasm. J Neurosurg 79: 490-493, 1993

3) Gourley JK, Heistad DD: Characteristics of reactive hyperemia in the cerebral circulation. Am J Physiol 246: H52-H58, 1984

4) Kuroki K, Taguchi H, Yukawa O: Hyperperfusion syndrome after clipping of an unruptured aneurysm: case report. Neurol Med Chir (Tokyo) 46: 248-250, 2006

5) Macfarlane R, Moskowitz MA, Sakas DE, Tasdemiroglu E, Wei EP, Kontos HA: The role of neuroeffector mechanisms in cerebral hyperperfusion syndromes. J Neurosurg 75: 845-855, 1991

6) Murakami H, Inaba M, Nakamura A, Ushioda T: Ipsilateral hyperperfusion after neck clipping of a giant internal carotid artery aneurysm. J Neurosurg 97: 1233-1236, 2002

7) Nedergaard $M$ : Mechanisms of brain damage in focal cerebral ischemia. Acta Neurol Scand 77: 81-101, 1988

8) Nicholas GG, Hashemi H, Gee W, Reed JF: The cerebral hyperperfusion syndrome: diagnostic value of ocular pneumoplethysmography. J Vasc Surg 17: 690-695, 1993

9) Ohta H: Postoperative luxury perfusion syndrome in patients with severe subarachnoid hemorrhage treated by early aneurysmal clipping. Neurol Med Chir (Tokyo) 30: 
16-23, 1990

10) Reigel MM, Hollier LH, Sundt TM, Piepgras DG, Sharbrough FW, Cherry KJ: Cerebral hyperperfusion syndrome: a cause of neurologic dysfunction after carotid endarterectomy. J Vasc Surg 5: 628-634, 1987

11) Sbarigia MA, Speziale F, Giannoni MF, Colonna M, Panico MA, Fiorani P: Post-carotid endarterectomy hyperperfusion syndrome: preliminary observations for identifying at risk patients by transcranial Doppler sonography and the acetazolamide test. Eur J Vasc Surg 7: 252-256, 1993

12) Takano T, Tian GF, Peng W, Lou N, Lovatt D, Hansen AJ, Kasischke KA, Nedergaard M: Cortical spreading depression causes and coincides with tissue hypoxia. Nat Neurosci
10: 754-762, 2007

13) Tomita M, Schiszler I, Tomita $Y$, Tanahashi N, Takeda $H$, Osada T, Suzuki N: Initial oligemia with capillary flow stop followed by hyperemia during $\mathrm{K}+$-induced cortical spreading depression in rats. J Cereb Blood Flow Metab 25: 742-747, 2005

Address reprint requests to: Toshiya Sugino, M.D., Department of Neurosurgery, Sapporo Medical University, South 1, West 16, Chuo-ku, Sapporo 060-8543, Japan.

e-mail: t-sugino@sapmed.ac.jp 